\title{
Tension Pneumothorax During Rigid Bronchoscopy for Chronic Foreign Body Removal in a Child: A Case Report
}

\author{
Akshay Kumar ${ }^{1}$, Nimisha Shiwalkar ${ }^{2}$, Hunain Aslam ${ }^{3}$, Purnadeo Persaud ${ }^{4}$ \\ 1. Cardiothoracic Surgery, Lokmanya Tilak Municipal Medical College \& General Hospital, Mumbai, IND 2. \\ Anesthesiology, Lokmanya Tilak Municipal Medical College \& General Hospital, Mumbai, IND 3. Surgery, University of \\ Missouri, Columbia, USA 4. Kansas City University of Medicine and Biosciences, Kansas City, USA
}

Corresponding author: Akshay Kumar, drakshay82@gmail.com

\begin{abstract}
Rigid bronchoscopy for chronic foreign body removal can cause rare but life-threatening complications in the form of tension pneumothorax. A two-year-old child who developed sudden cardiac arrest during the procedure required urgent chest tube insertion. Integrated team effort with effective communication prevented devastating neurological sequelae from hypoxic ischemic encephalopathy.
\end{abstract}

Received 07/18/2019

Review began 09/03/2019

Review ended 09/09/2019

Published 09/11/2019

○ Copyright 2019

Kumar et al. This is an open access article distributed under the terms of the Creative Commons Attribution License CC-BY 3.0., which permits unrestricted use, distribution, and reproduction in any medium, provided the original author and source are credited.
Categories: Anesthesiology, Medical Education, Pediatric Surgery

Keywords: respiratory, resuscitation, imaging, intensive care, tension pneumothorax, tracheobronchial foreign body, rigid bronchoscopy, intercostal tube

\section{Introduction}

Bronchoscopy was developed and first used in 1895 for removing foreign bodies from the main stem bronchi, when Gustav Killian removed a piece of bone from the right main-stem bronchus of a 36-year-old man [1]. Performing these procedures in airway of pediatric patients poses a special challenge to both the anesthesiologist and the surgeon. Pneumothorax remains a rare but devastating complication instigating from lower airway manipulation during rigid bronchoscopy [2]. Rothmana and Boeckman reported that the probability of developing pneumothorax during rigid bronchoscopy for the removal of foreign body is approximately one in 100 cases [3]. The possibility of tension pneumothorax should be borne in mind in a mechanically ventilated patient with persistently rising airway pressures and progressively diminishing cardiac output. We herein report a challenging case of foreign body removal, where the patient developed cardiac arrest due to intraoperative pneumothorax and was revived with continued successful cardiopulmonary resuscitation. In the particular case of the patient being a minor, a written informed parental consent was taken to publish this case report.

\section{Case Presentation}

A two-year-old male child, weighing $15 \mathrm{~kg}$, was presented to the outpatient clinic with complaints of recurrent lower respiratory tract infections for one year. Air entry was decreased in the left lower zone. Rest of respiratory as well as cardiac and neurological examinations were within normal limits. Chest X-ray revealed emphysematous changes on the right side and shift of mediastinum to the left (Figure 1). 


\section{Cureus}

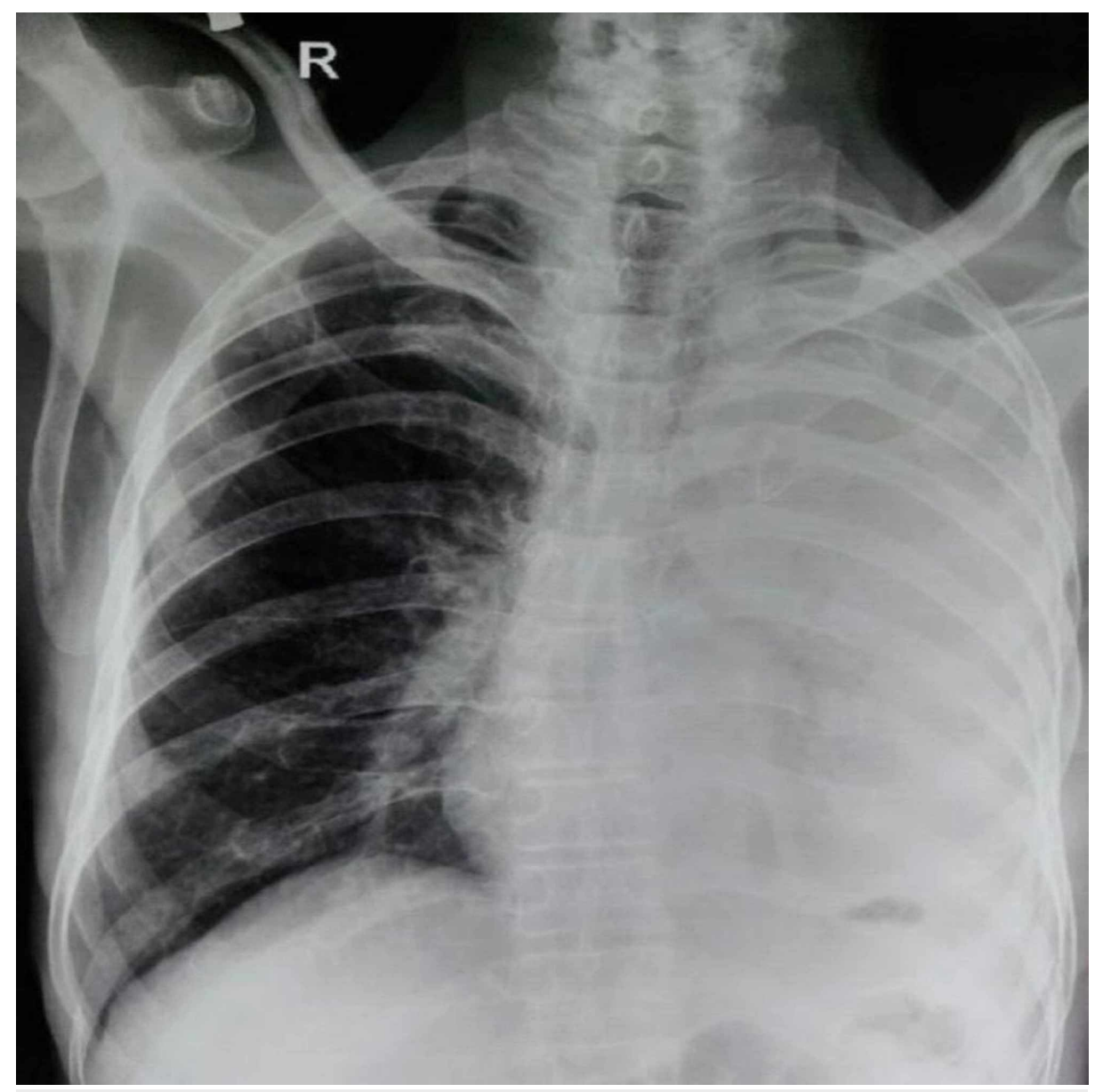

\section{FIGURE 1: Chest X-ray showing haziness in the left chest and emphysematous changes in the right side of the chest}

Contrast-enhanced CT scan revealed focal stenosis of left lower lobe bronchus due to possible foreign body or bronchial stricture, and further evaluation with bronchoscopy was recommended. Thus, the patient was taken up for rigid bronchoscopic evaluation for removal of foreign body under general anesthesia with controlled manual ventilation. The patient was premedicated with intravenous Inj glycopyrrolate $0.06 \mathrm{mg}$, Inj midazolam $0.3 \mathrm{mg}$, Inj ranitidine $15 \mathrm{mg}$, Inj ondansetron $1.5 \mathrm{mg}$, and Inj fentanyl $30 \mu \mathrm{g}$. The patient was then induced with Inj propofol in graded doses till loss of consciousness followed by Inj suxamethonium 1 $\mathrm{mg} / \mathrm{kg}$. Rigid bronchoscope was then introduced, and anesthesia was maintained through the side port of the bronchoscope with $100 \% \mathrm{O}_{2}$, sevoflurane, and intermittent atracurium. Bronchoscopy revealed foreign body in the left lower bronchus at its take off from the left main stem bronchus. Subsequently, multiple attempts were made to remove the foreign body using graspers (Figure 2). 


\section{Cureus}

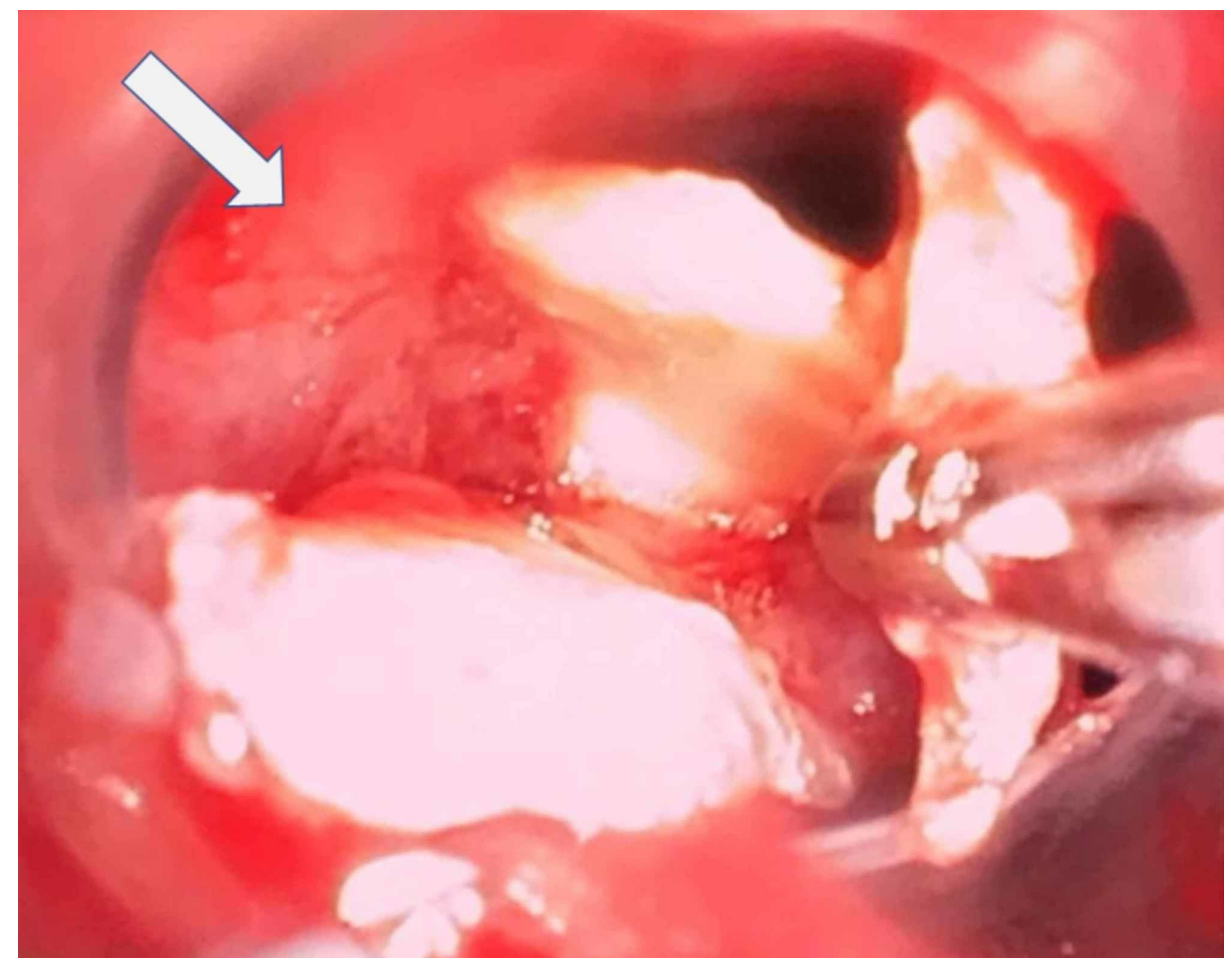

FIGURE 2: Intraoperative picture of bronchoscopy showing foreign body being retrieved by grasper

After about 30 minutes into the procedure, the patient suddenly developed bradycardia and dropped his saturations to low 70s culminating in asystole. Cardiopulmonary resuscitation (CPR) was initiated immediately as per the PALS (Pediatric Advanced Life Support) protocol. Simultaneously, a search for cause for cardiac arrest revealed pneumothorax on the left side. Immediately intercostal drainage was inserted in left chest in the fifth intercostal space in the anterior axillary line (Figure 3). 


\section{Cureus}

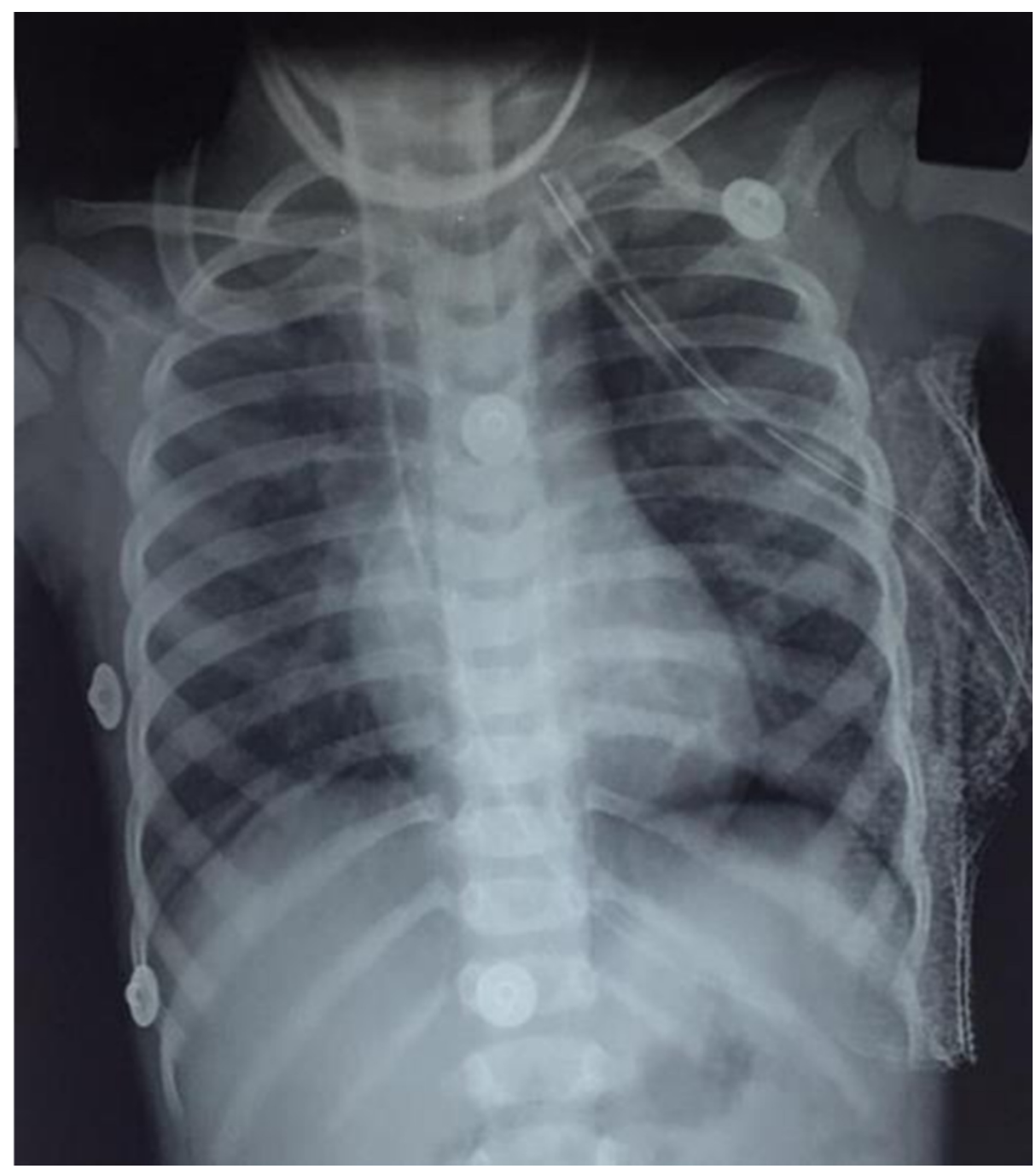

FIGURE 3: Intraoperative chest X-ray after insertion of intercostal drainage tube

The child was shifted to the intensive care unit in hemodynamically stable (hazard ratio-104/min, blood pressure-94/62 $\mathrm{mmHg}, \mathrm{SPO}_{2}-95 \%$ ) but guarded condition. The hemodynamic profile of the child in the pre-, intra-, and postoperative period after resuscitation is shown in Table 1 . On postoperative day, one patient woke up and did not show any signs of respiratory or neurological deficit and was extubated. There was no air leak or residual pneumothorax, and his chest tube was removed on day two. His postoperative recovery was uneventful and was discharged from hospital after few days. 


\begin{tabular}{|c|c|c|c|c|c|c|c|c|}
\hline & & Preop & Intraop & Intraoperative (30 min) & $\begin{array}{l}\text { Prior to } \\
\text { Arrest }\end{array}$ & $\begin{array}{l}\text { Post Chest tube } \\
\text { Insertion }\end{array}$ & Postop & In ICU \\
\hline Temp (Celsius) & & 37.4 & 37.4 & $3 / .5$ & $3 / .2$ & 37.0 & $3 / .8$ & 37.4 \\
\hline HR (per minute) & & 94 & 98 & 64 & 40 & 140 & 118 & 104 \\
\hline $\begin{array}{l}\text { BP (syst/diastolic) } \\
\text { (mmHg) }\end{array}$ & Mean & $\begin{array}{l}88 / 58 \\
(68)\end{array}$ & $\begin{array}{l}82 / 56 \\
(55)\end{array}$ & $\begin{array}{l}68 / 48 \\
(62)\end{array}$ & $\cdots$ & $58 / 38(50)$ & $\begin{array}{l}84 / 58 \\
(68)\end{array}$ & $\begin{array}{l}94 / 62 \\
(104 / 84)\end{array}$ \\
\hline $\mathrm{SpO}_{2}$ & & 99 & 99 & 72 & 34 & 94 & 92 & 95 \\
\hline
\end{tabular}

TABLE 1: Hemodynamic profile of the child in the preoperative and intraoperative during rigid bronchoscopy, prior to arrest, post chest tube insertion, and postoperative in the ICU

BP, blood pressure; HR, hazard ratio; ICU, intensive care unit.

\section{Discussion}

Rigid bronchoscopy for retrieval of foreign bodies is a common procedure in pediatric age group, though it is a very challenging case scenario. Complications include bleeding, infection, laryngeal edema, laryngospasm, bronchospasm, hypoxemia, airway injury with tracheobronchial laceration, and pneumothorax usually resulting from direct trauma to tracheobronchial tree $[4,5]$. Though the possibility of pneumothorax is less than one percent, it remains one of the dreaded complications during bronchoscopy [6]. The addition of positive pressure due to high-frequency jet ventilation converts pneumothorax into a tension pneumothorax. This occurs when one-way valve mechanism develops because of tracheo-bronchial instrumentation leading inadvertent rent in airway, and thereby allowing the air to enter the pleural cavity during positive pressure ventilation and preventing exit during expiratory phase. As the air builds up in the pleural space, ipsilateral lung is compressed followed by mediastinal shift and compression of contralateral lung and intrathoracic vasculature leading to severe hypoxemia and cardiovascular compromise [7]. The development of tension is dependent on a pressure gradient between intrapleural pressure (IPP) and alveolar pressure. Continued ventilation increases gas flow through the pleural defect, allowing more air to pass per unit time. This accentuates the rise in IPP with earlier mechanical compressive effects and rapid progress to cardiorespiratory collapse [8-10]. Early and prompt diagnosis of pneumothorax during anesthesia, however, can be tricky as the symptoms may be masked due to anesthesia in a mechanically ventilated patient [11]. Diagnosis in these patients rests upon high index of suspicion and recognition of supportive diagnostic features. A sudden fall in $\mathrm{SPO}_{2}$ ensues followed by hypotension (over a few minutes) as was noted in Steier's large case series and is consistently found in the individual case reports [12]. Progression to tension pneumothorax can lead to a rapid increase in airway pressures culminating in bradycardia and cardio respiratory arrest. Because of this sudden nature of presentation, tension pneumothorax is more often than likely to be missed in ventilated patients [13]. A low threshold for performing tube or finger thoracostomy must be maintained in mechanically ventilated patients [14]. This reiterates that continuous vigilant monitoring is essential to alert the anesthesiologist of a worsening situation.Hussain et al. reported a one-year-old female child developing tension pneumothorax following rigid bronchoscopy for acute foreign body aspiration [15]. In more grievous injury to the tracheo-bronchial tree requiring surgical repair or airway reconstruction, it is prudent to place the child on extracorporeal circulatory support, repair the injury, and provide lung protective ventilation [16]. To the best of our knowledge, this is the first case reported in the literature for tension pneumothorax complicated by cardiac arrest following rigid bronchoscopy for chronic foreign body removal in such a small child managed successfully.

\section{Conclusions}

A high index of suspicion must be maintained for pneumothorax during rigid bronchoscopy whenever sudden deterioration of hemodynamic or hypoxia ensues. Intraoperative catastrophe from which a child cannot be revived or suffers irreversible neurological damage can hamper the morale of the healthcare team. An integrated teamwork emphasizing on efficient communication and coordination between the surgeon, anesthesiologist, scrub nurse, circulating nurse, technicians, and perfusionist is of utmost importance in saving the child's life.

\section{Additional Information \\ Disclosures}

Human subjects: Consent was obtained by all participants in this study. Conflicts of interest: In compliance with the ICMJE uniform disclosure form, all authors declare the following: Payment/services 
info: All authors have declared that no financial support was received from any organization for the submitted work. Financial relationships: All authors have declared that they have no financial relationships at present or within the previous three years with any organizations that might have an interest in the submitted work. Other relationships: All authors have declared that there are no other relationships or activities that could appear to have influenced the submitted work.

\section{References}

1. Beamis JF: Modern use of rigid bronchoscopy . Interventional Bronchoscopy . Karger, New York; 2000.

2. Harar RPS, Pratap R, Chadha N, Tolley N: Bilateral tension pneumothorax following rigid bronchoscopy: a report of an epignathus in a newborn delivered by the EXIT procedure with a fatal outcome. J Laryngol Otol. 2005, 119:400-402. 10.1258/0022215053945813

3. Coats TJ, Wilson AW, Xeropotamous N: Pre-hospital management of patients with severe thoracic injury . Injury. 1995, 26:581-585. 10.1016/0020-1383(95)00107-K

4. Hasdiraz L, Oguzkaya F, Bilgin M, Bicer C: Complications of bronchoscopy for foreign body removal: experience in 1,035 cases. Ann Saudi Med. 2006, 26:283-287. 10.5144/0256-4947.2006.283

5. Steen KH, Zimmermann T: Tracheobronchial aspiration of foreign bodies in children: a study of 94 cases . Laryngoscope. 1990, 100:525-530. 10.1288/00005537-199005000-00016

6. Ibrahim AE, Stanwood PL, Freund P: Pneumothorax and systemic air embolism during positive-pressure ventilation. Anesthesiology. 1999, 90:1479-1481. 10.1097/00000542-199905000-00035

7. Brian H, Martin LM: Pleural disorders in the intensive care units. Washington Manual of Critical Care. Marin Kollef (ed): Lippincott Williams \& Wilkins, Philadelphia; 2017.

8. Loer S, Fritz KW: Life threatening tension pneumothorax after puncture of the subclavian vein and dislocation of thoracic drainage. Anaesthesiol Reanim. 1994, 19:137-138.

9. Mariani PJ, Sharma S: Iatrogenic tension pneumothorax complicating outpatient Heimlich valve chest drainage. J Emerg Med. 1994, 12:477-479. 10.1016/0736-4679(94)90343-3

10. Tan EC, Van der Vliet JA: Delayed (tension) pneumothorax after placement of a central venous catheter. Ned Tijdschr Geneeskd. 1999, 143:1872-1875.

11. Baumann MH, Sahn SA: Tension pneumothorax: diagnostic and therapeutic pitfalls . Crit Care Med. 1993, 21:177-179.

12. Deakin CD, Davies G, Wilson A: Simple thoracostomy avoids chest drain insertion in prehospital trauma . J Trauma Acute Care Surg. 1995, 39:373-374. 10.1097/00005373-199508000-00031

13. Tocino IM, Miller MH, Fairfax WR: Distribution of pneumothorax in the supine and semirecumbent critically ill adult. AJR Am J Roentgenol. 1985, 144:901-905. 10.2214/ajr.144.5.901

14. Schmidt U, Stalp M, Gerich T, Blauth M, Maull KI, Tscherne H: Chest tube decompression of blunt chest injuries by physicians in the field: effectiveness and complications. J Trauma. 1998, 44:98-100. 10.1097/00005373-199801000-00010

15. Hussain S, Khan RA, Iqbal M: Tension pneumothorax caused by ventilating rigid bronchoscopy for removal of foreign body. Anaesth Crit Care Pain Med. 2011, 15:57-59.

16. Han KA, Kim HJ, Byon HJ, et al.: Cardiac arrest induced by tension pneumothorax during ventilating bronchoscopy: a case report. Korean J Anesthesiol. 2010, 59:123-126. 10.4097/kjae.2010.59.2.123 